\title{
Aneurysmal Bone Cyst and Pregnancy: Is There Any Association?
}

\author{
Ravishankar S. Goel ${ }^{1} \quad$ Devendra Purohit $^{1} \quad$ Jitendra Sekhawat $^{1} \quad$ R. S. Mittal ${ }^{1}$ \\ ${ }^{1}$ Department of Neurosurgery, Sawai Man Singh Hospital, Jaipur, \\ Rajasthan, India \\ Indian J Neurosurg 2015;4:185-189.

\begin{abstract}
Address for correspondence Ravishankar S. Goel, MCh, Department of Neurosurgery, Sawai Man Singh Hospital, Jaipur, Rajasthan 302004, India (e-mail: docravishankar@yahoo.co.in).
\end{abstract}

\author{
Abstract \\ Keywords \\ - aneurysmal bone cyst \\ - vascular tumor \\ - pregnancy \\ - pathological fracture \\ - spinal cord \\ compression
}

Aneurysmal bone cyst $(A B C)$ is an expansile, osteolytic lesion. The association of $A B C$ with pregnancy is a very rare entity. We are reporting a unique case of spinal $A B C$, presenting as pathological fracture, paraplegia, and abdominal mass in the 28th week of pregnancy. A brief review of the literature is discussed. Dyshomeostasis of bone calcium, hormonal, and mechanical stress can aggravate the severity of $A B C$ during pregnancy. A multidisciplinary approach is required to manage such cases, depending on the fetal gestational age and maternal neurological features.

\section{Introduction}

The World Health Organization defined aneurysmal bone cyst $(A B C)$ as "an expanding lesion with blood-filled cavities, separated by septa of trabecular bone or fibrous tissuecontaining osteoclast giant cells." It is usually found in the long bones and develops before the second decade of life. ${ }^{2}$ Incidence of spinal $A B C$ is $15 \%$ of all primary spine tumors. ${ }^{1}$ Association of $A B C$ with pregnancy is a very rare entity. ${ }^{2}$ It may enlarge during pregnancy. ${ }^{2}$ We are reporting a unique case of aneurysmal bone cyst of L2 vertebra, presenting as pathological fracture, paraplegia, and retroperitoneal mass during pregnancy.

\section{Case Report}

A 26-year-old pregnant (28 week) female patient was admitted in the obstetrics department with paraplegia with fetal distress. She had a history of low backache for 10 days. Immediately a premature baby was delivered by cesarean section. She was referred to the neurosurgery department for further evaluation. On examination, hypotonia was present in both the lower limbs. Power in both lower limbs were 0/5 (the Medical Research Council grade) at the hips, knee, ankles, and great toe for all movements. Superficial abdominal reflexes were present.
Plantar reflexes were bilaterally mute. There was a $20 \%$ sensory deficit below L1 level bilaterally for all modalities. There was no bowel, bladder disturbances.

Computed tomography scan lumbosacral (LS) spine showed an L2 vertebral body fracture with a left paraspinal mass, involving L2 vertebra (-Fig. 1A1-A3) and magnetic resonance imaging (MRI) showed large expansile multiloculated lesion, with retroperitoneal extension with fluid-fluid levels with $\mathrm{L} 2$ vertebral body fracture suggestive of aneurysmal bone cyst ( - Fig. 2A1-A3). After discussion of risk factors with relatives, surgery was planned. Preoperative embolization was done with polyvinyl aspartate particles $(150-200 \mu \mathrm{m})$ one day before surgery. L1, L2 laminectomy, removal of intraspinal portion of $A B C$ (-Fig. 3A, B) and D12 to L3 pedicle screw fixation was done. After 1 month, complete excision of retroperitoneal part was done, with the help of the general surgeon's team, through transabdominal approach. The histopathological examination had confirmed $A B C$ (-Fig. 4A, B). Immunohistochemistry was estrogen and progesterone receptor negative (ER/PR) and Her-2/neu negative. Postoperative X-ray LS spine showed pedicle screw in situ (-Fig. 1B1, B2). Postoperative MRI showed residual ABC after the first surgery ( - Fig. 2B1-B3) and complete excision of $A B C$ after second surgery ( - Fig. 2C1-C3). Postoperative period was uneventful with significant recovery. received

April 7, 2015

accepted

September 22, 2015

published online

December 16, 2015
DOI http://dx.doi.org/

10.1055/s-0035-1569000. ISSN 2277-954X.
(C) 2015 Neurological Surgeons' Society of India
License terms

(요 (1) $\Theta$ 


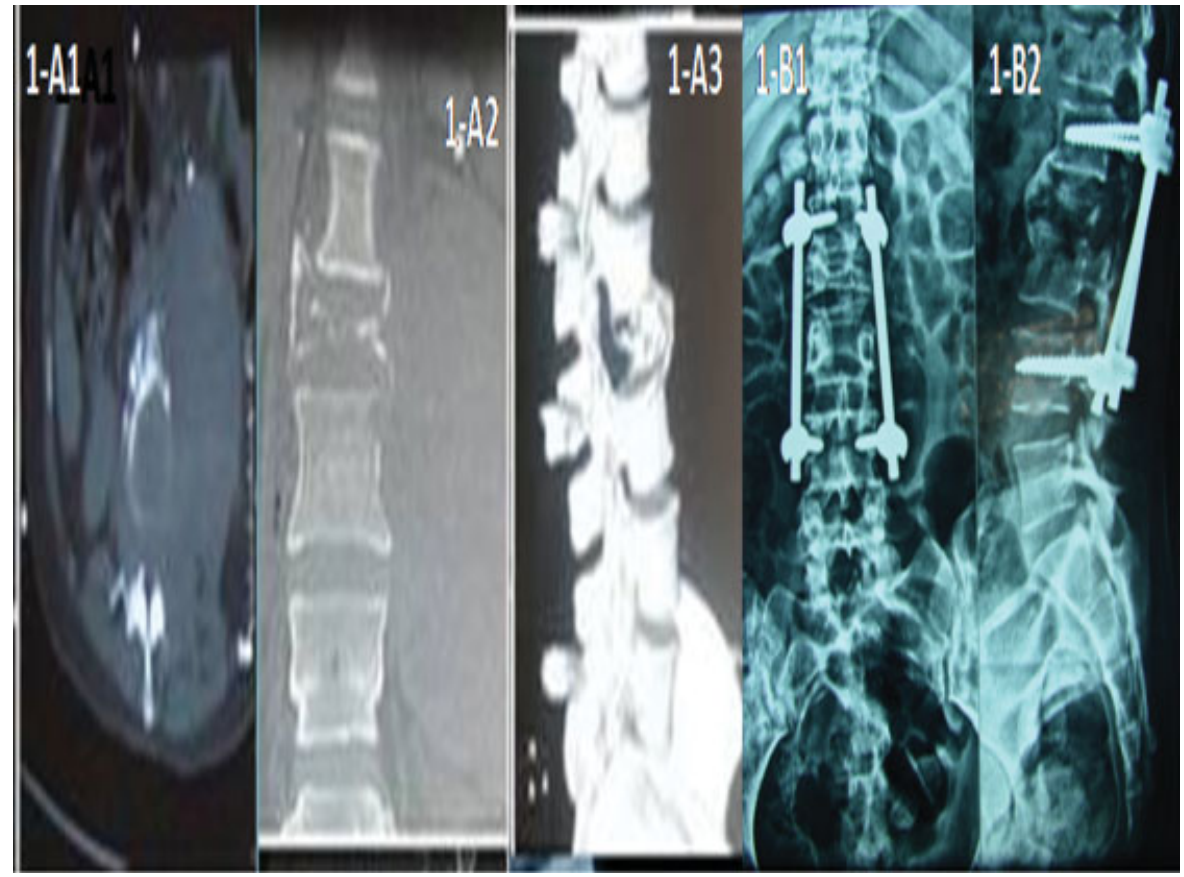

Fig. 1 (A) Preoperative CT scan LS spine (A1: axial, A2: coronal, A3: sagittal with 3D reconstruction) showing L2 vertebral body fracture with a left paraspinal mass (B1, B2). (B) Postoperative X-ray LS spine (B1: anteroposterior, B2: lateral view) showing pedicle screw in situ. CT, computed tomographic; LS, lumbosacral.

At 3 months follow-up, power was $4 / 5$ at the hips, knee, and great toe and $3 / 5$ at the ankle. There were no sensory deficit and no bowel, bladder disturbance. The patient was able to walk with support. As the gestational effect could not be predicted, she was advised against next pregnancy. Relatives were explained the need of radiotherapy, if recur.

\section{Discussion}

ABCs can occur as primary $(70 \%)$ or secondary $(30 \%)$ lesion. ${ }^{3}$ Primary $A B C$ is considered as neoplasm, driven by upregulation of the ubiquitin specific protease USP6 (Tre2) gene. ${ }^{3}$ Secondary $\mathrm{ABCs}$ are associated with other lesions. Pathogenesis of enlargement of $A B C$ during pregnancy is not clear. $^{2}$ According to Webber et al, ABCs associated with pregnancy might be primary neoplasm as USP 6 positive. ${ }^{4}$ However, it also consists of reactive component explained on the basis of the presence of insulin-like growth factor-1 (IGF-1) receptor expression. ${ }^{4}$

Few cases of the $A B C s$ are reported with pregnancy $2,4-10$ (-Table 1). Change in symptomatology can be due to hormonal changes during pregnancy. ${ }^{2,4,7,9}$ Cataltepe et al proposed hormonal changes, such as increased hematopoietic activity, increased cortisone, with vitamin D antagonistic effect, and human placental lactogen with growth hormone-like effect as a causative factor in pregnancy. ${ }^{2}$ Webber et al suggested increased IGF-1, involved in bone remodeling and repair, as a causative factor during pregnancy. ${ }^{4}$ Amanatullah et al suggested role of circulating growth hormone in pregnancy, along with transforming growth receptor $-\beta$ receptors on cells of $A B C{ }^{9}$
Progesterone and estrogen levels increase during pregnancy and causes venous distension and vascular growth, respectively, which can increase local venous pressure. Parathyroid hormone-related protein also increases during pregnancy and can cause osteoporosis and pathological fracture. Human placental growth factors (HPIGF), which are involved in bone remodeling and repair, can be a causative factor in pregnancy. In the present case of $\mathrm{ABC}$, staining for $\mathrm{ER}, \mathrm{PR}$, and Her-2/neu were negative. In three cases, reported by Webber et al, ${ }^{9}$ staining for ER, PR, and HPIGF were negative, but positive for IGF- $1 .{ }^{4}$

Gravid uterus increases the pressure in paravertebral veins by compressing the vena cava, which causes hemorrhage and osteolysis, further leading to hemorrhage and amplification of $A B C s$.

Management of $\mathrm{ABC}$ in third trimester includes cesarean section, followed by spine fixation. Expulsive forces of labor could predispose to life-threatening uncontrolled bleeding. Total excision is the treatment of choice, if feasible. Curettage and bone grafting are the standard treatment for the central spinal $A B C$. If there is vertebral body involvement, combined anterior and posterior approaches for tumor removal and fusion are required to maintain the structural integrity.

Radiotherapy is helpful to diminish the blood supply of the tumor when complete surgical excision is not possible. Recent studies showed that denosumab, a human monoclonal antibody can treat $\mathrm{ABCs}{ }^{4}$ In the future, denosumab can play a major role in treatment policies.

Prognosis is good, even after recurrence. Recurrence after surgery is related to the cavities not been fully opened. A residual small cyst with radiolucency is of concern and 

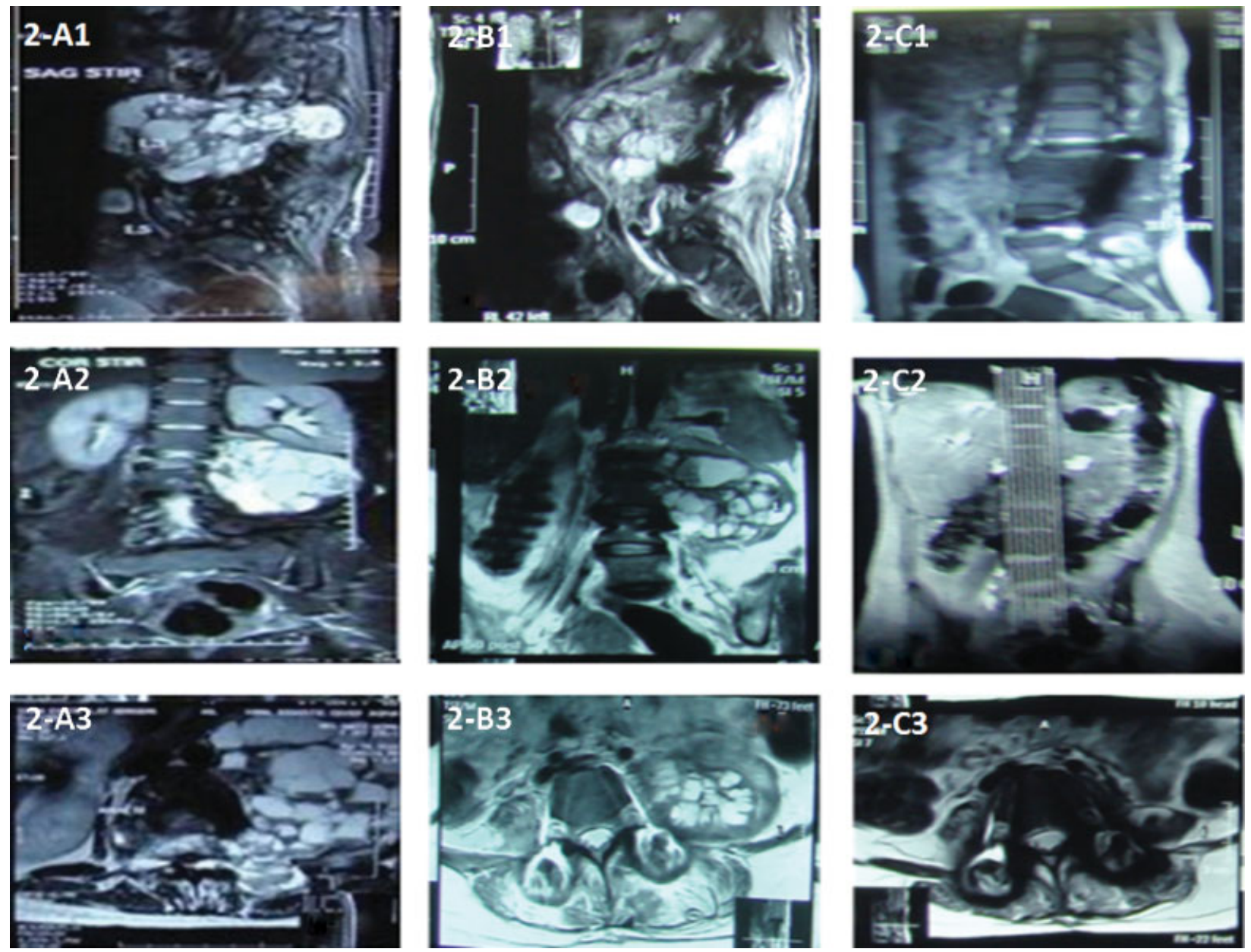

Fig. 2 MRI DL spine. (A) Preoperative (A1: sagittal, A2: coronal, A3: axial section) showing large expansile multiloculated lesion, with retroperitoneal extension with fluid-fluid levels with $L 2$ vertebral body fracture. (B) Postoperative after first surgery (B1: sagittal, B2: coronal, B3: axial section) showing residual $A B C$. (C) After second surgery (C1: sagittal, $C 2$ : coronal, $C 3$ : axial section) showing complete removal of $A B C$. $\mathrm{DL}$, degenerative lumbar; MRI, magnetic resonance imaging.
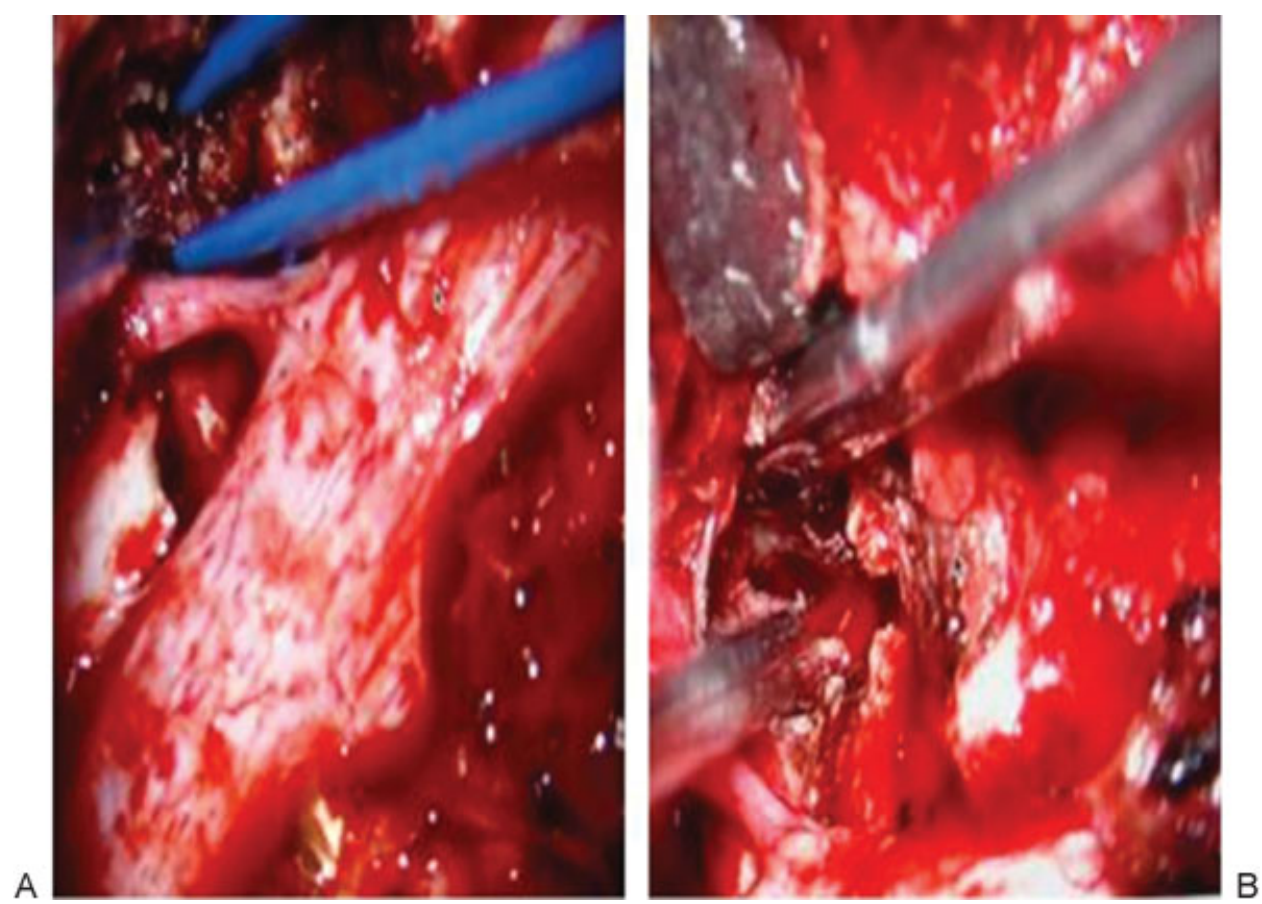

Fig. 3 (A, B) Intraoperative photograph showing posterior decompression. 

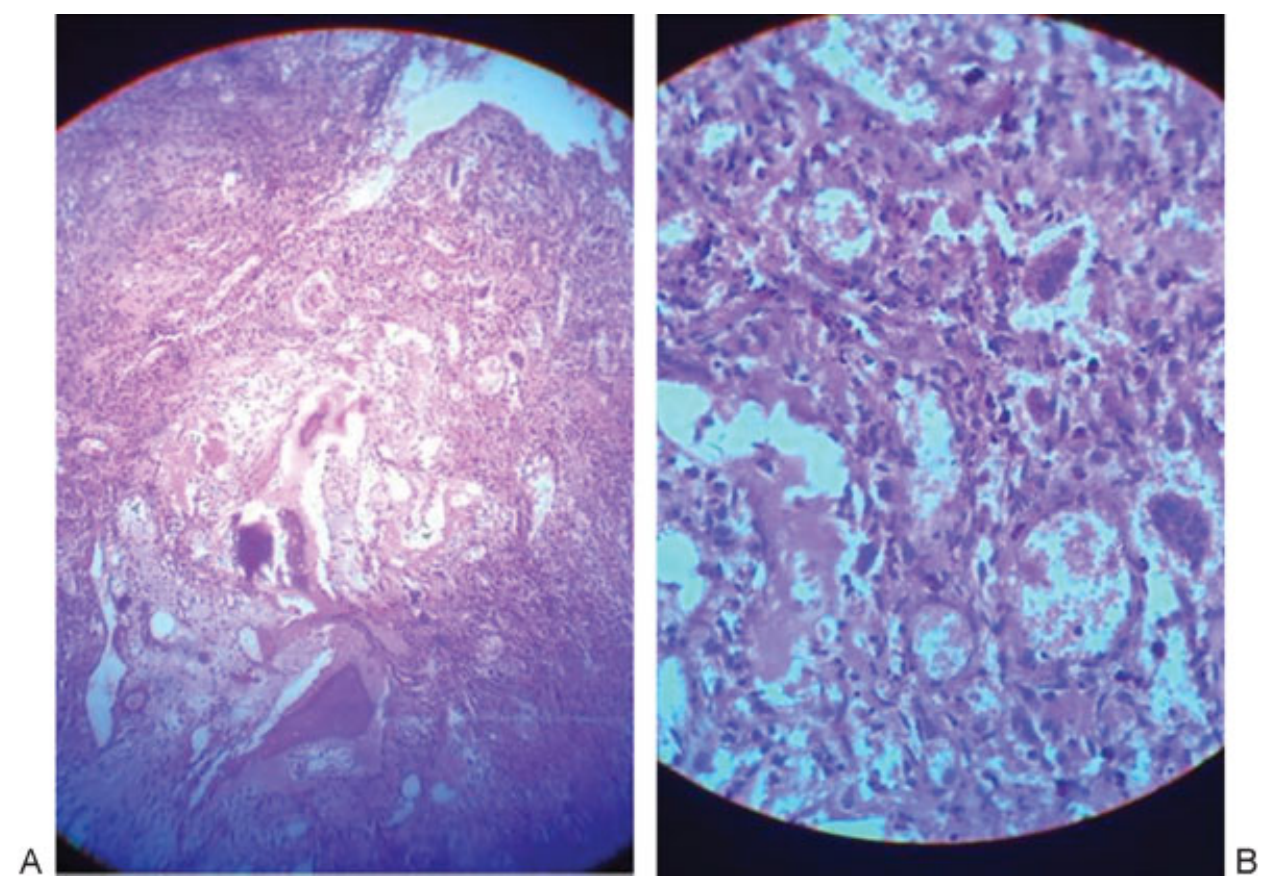

Fig. 4 Microphotographs of histopathological examination (hematoxylin \& eosin staining (A) $\times 10$ and (B) $\times 40$ ) multiple blood-filled lacunae, separated by collagenous tissue containing spindle-shaped fibroblasts, dilated capillaries, and osteoclastic giant cells, with no endothelial lining.

Table 1 Reported cases of aneurysmal bone cyst during pregnancy

\begin{tabular}{|c|c|c|c|c|c|}
\hline Serial number & Author & Year & Number of cases & Site & Primary vs. secondary \\
\hline 1 & Baker et $\mathrm{al}^{5}$ & 1982 & 1 & Ethmoid & Primary \\
\hline 2 & Issa et $\mathrm{al}^{6}$ & 1986 & 1 & Right ilium & Primary \\
\hline 3 & Mintz et $\mathrm{al}^{7}$ & 1987 & 1 & $\begin{array}{l}\text { Right humerus and acromian } \\
\text { process of left scapula }\end{array}$ & Secondary \\
\hline 4 & Cataltepe et $\mathrm{al}^{2}$ & 1990 & 1 & Frontal bone & Primary \\
\hline \multirow[t]{3}{*}{5} & \multirow[t]{3}{*}{ Webber et $\mathrm{al}^{4}$} & \multirow[t]{3}{*}{2011} & \multirow[t]{3}{*}{3} & Femur & Primary \\
\hline & & & & Scapula & Primary \\
\hline & & & & Humerus & Primary \\
\hline 6 & Westbury et al ${ }^{8}$ & 2011 & 1 & Mandible & Secondary \\
\hline 7 & Amanatullah et $\mathrm{al}^{9}$ & 2012 & 1 & Humerus & Primary \\
\hline 8 & Elkattah et al ${ }^{10}$ & 2013 & 1 & Left ilium & Primary \\
\hline 9 & Present case & 2014 & 1 & Lumbar spine & Primary \\
\hline
\end{tabular}

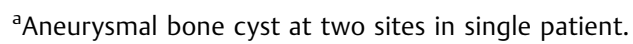

requires regular follow-up. Aneurysmal bone cyst can increase in size during pregnancy. Timely diagnosis can avoid complications.

\section{References}

1 Burch S, Hu S, Berven S. Aneurysmal bone cysts of the spine. Neurosurg Clin N Am 2008;19(1):41-47
2 Cataltepe O, Inci S, Ozcan OE, Sağlam S, Erbengi A. Aneurysmal bone cyst of the frontal bone. Surg Neurol 1990;33(6):391-394

3 Pauli C, Fuchs B, Pfirrmann C, Bridge JA, Hofer S, Bode B. Response of an aggressive periosteal aneurysmal bone cyst $(\mathrm{ABC})$ of the radius to denosumab therapy. World J Surg Oncol 2014;12:17

4 Webber NP, Grossmann AH, Tripp S, et al. Clinical, molecular, cytogenetic, and immunohistochemical features of pregnancyassociated aneurysmal bone cyst. Poster presented at: 63rd Annual Meeting of Association of Bone and Joint Surgery; June 812, 2011; Dublin, Ireland 
5 Baker HL, Papsidero MJ, Batsakis JG, Krause CJ. Aneurysmal bone cyst of the ethmoid. Head Neck Surg 1982;5(2): $177-180$

6 Issa AA, Amr SS, Swaiss AM. Aneurysmal bone cyst of the ilium associated with pregnancy. Eur J Obstet Gynecol Reprod Biol 1986;23(3-4):243-248

7 Mintz MC, Dalinka MK, Schmidt R. Aneurysmal bone cyst arising in fibrous dysplasia during pregnancy. Radiology 1987;165(2): $549-550$
8 Westbury SK, Eley KA, Althanosou N, Anand R, Watt-Smith SR. Giant cell granuloma with aneurysmal bone cyst change within the mandible during pregnancy: a management dilemma. J Oral Maxillofac Surg 2011;69(4):1108-10133

9 Amanatullah DF, Bozzio AE, Mallen ZO, Mak WH, Borys D, Tamurian RM. Lateral elbow pain in 21 year old pregnant female. Shoulder Elbow 2012;4(1):46-49

10 Elkattah R, Foulk B. A suspected pelvic aneurysmal bone cyst in pregnancy. Case Rep Obstet Gynecol 2013;2013: 676087 